ISSN 0103-5150

Fisioter. Mov., Curitiba, v. 26, n. 1, p. 151-158, jan./mar. 2013

Licenciado sob uma Licença Creative Commons

\title{
Influência das orientações fisioterapêuticas na qualidade de vida e na sobrecarga de cuidadores
}

\author{
Influence of physiotherapeutic guidelines in the \\ caregiver's quality of life and overload
}

\author{
Bruna Cristina Warken Fernandes ${ }^{[a]}$, Karla Caroline Pacheco Ferreira ${ }^{[a]}$, Maira Franciele Marodin ${ }^{[a]}$, \\ Maria Odette Nassif do Val ${ }^{[b]}$, Andersom Ricardo Fréz ${ }^{[c]}$ \\ [a] Fisioterapeutas pela Faculdade Anglo-Americano (FAA), Foz do Iguaçu, PR - Brasil, e-mails: brunawarken@hotmail.com, \\ kaka_karlinha@hotmail.com,mahmarodin@hotmail.com \\ [b] Fisioterapeuta, especialista em Fisioterapia Neurofuncional pela Universidade Norte do Paraná (Unopar), docente da \\ Faculdade Anglo-Americano (FAA), Foz do Iguaçu, PR - Brasil, e-mail: cerenemo@hotmail.com \\ [c] Fisioterapeuta, mestre em Saúde, Interdisciplinaridade e Reabilitação pela Universidade Estadual de Campinas \\ (Unicamp), docente da Universidade Estadual do Centro-Oeste (Unicentro), Guarapuava, PR - Brasil, e-mail: \\ andersom_frez@yahoo.com.br
}

\section{Resumo}

Introdução: 0 despreparo do cuidador pode acarretar situações de risco para quem cuida e para quem recebe o cuidado. Objetivo: Avaliar a influência de orientações fisioterapêuticas na qualidade de vida e na sobrecarga de cuidadores informais de pacientes com disfunções decorrentes de acidente vascular encefálico (AVE) ou de trauma raquimedular (TRM). Materiais e métodos: Doze cuidadoras informais, com idade média de 50,5 \pm 16,5 anos, sendo 4 de pacientes com disfunções decorrentes de TRM e 8 de pacientes que sofreram AVE. Todos os pacientes estavam em atendimento fisioterapêutico. No primeiro encontro, as cuidadoras foram instruídas a anotar as dúvidas e dificuldades do dia a dia. Após uma semana, entregaram as anotações; na mesma ocasião, foi avaliada a qualidade de vida e a sobrecarga, utilizando o questionário SF-36 e o Questionário de Avaliação da Sobrecarga do Cuidador Informal, respectivamente. Duas semanas depois, foi realizada uma aula teórico-prática baseada nas dúvidas e nas respostas dos questionários. Após quatro semanas, foram reavaliadas. Para comparação pré e pós-intervenção, foi utilizado o teste $t$ pareado ( $p<0,05)$. Resultados: As dúvidas anotadas estavam relacionadas ao banho do paciente e com a própria saúde física e mental. Não se observou incremento no índice geral da qualidade de vida $(p=0,1226)$. Apenas 
para os domínios dor ( $\mathrm{p}=0,0316)$ e saúde mental $(\mathrm{p}=0,0029)$ observou-se diferença significativa. Não se observou diferença na sobrecarga do cuidador. Conclusão: Uma intervenção com orientações fisioterapêuticas repercutiu na redução da dor e melhora da saúde mental em cuidadores informais de pessoas com disfunções decorrentes de TRM ou AVE.

Palavra-chaves: Cuidadores. Educação em saúde. Qualidade de vida. Nível de saúde.

\section{Abstract}

Introduction: The caregiver's unpreparedness can lead to risky situations for caregivers and for those who receive the care. Objective: To assess the impact of the physiotherapeutic guidelines on quality of life and overload of informal caregivers of patients with spinal cord injury or stroke disabilities. Materials and methods: Twelve female informal caregivers, mean age 50,5 $\pm 16,5$ years old. Four were caregiver from patients with disability caused by spinal cord injury and 8 by stroke. All patients were in physical therapy. At the first meeting, the caregivers received a notepad to write down their daily difficulties. After a week, they returned the notepads. On this day we assessed the quality of life, using the SF-36, and the overload, using the Questionnaire for Assessment to Informal Caregiver Overload. Two weeks later, we prepared a theoretical and practical class based on the notes and the questionnaires scores. After 4 weeks, the caregivers were assessed again using the baseline instruments. To compare pre and post intervention was used paired $t$-test $(p \leq 0,05)$. Results: The doubts were about the patient's bath and their own physical and mental health. There was no increase in quality of life $(p=0.1226)$. Only for the domains of pain $(p=0.0316)$ and mental health $(p=0.0029)$ was observed a significant difference. There was no difference in caregiver overload. Conclusions: An intervention with physical therapy guidelines reduced pain and improved mental health in informal caregivers of patients with spinal cord injury or stroke disabilities.

Keywords: Caregivers. Health education. Quality of life. Health status.

\section{Introdução}

A Classificação Brasileira de Ocupações (CBO) define o cuidador como alguém que "cuida a partir dos objetivos estabelecidos por instituições especializadas ou responsáveis diretos, zelando pelo bem-estar, saúde, alimentação, higiene pessoal, educação, cultura, recreação e lazer da pessoa assistida" (1). Porém, sabe-se que muitos desses cuidados são realizados por cuidadores informais, que são familiares ou pessoas da comunidade sem qualquer formação para exercer tal atividade. Esse despreparo pode acarretar em ansiedade e desgaste físico, ocasionando situação de risco para quem cuida e para quem recebe o cuidado. Por isso, é necessário que o cuidador obtenha conhecimento suficiente sobre a patologia e sobre as estratégias que devem ser usadas no cuidar (2), pois obtendo orientações necessárias e fundamentais, o cuidador passa a ter mais garantia de suas ações, dando segurança para si mesmo e para o cuidado (3).

Muitas vezes, apenas uma pessoa fica encarregada de realizar todos os cuidados do paciente; quanto maior o nível de dependência, maior é o esforço da parte do cuidador, e este acaba deixando sua própria vida em segundo plano, o que implica em grande sobrecarga (2). Essa sobrecarga também pode ser resultado da combinação do esforço físico, da pressão emocional, das limitações sociais e das exigências econômicas que surgem ao cuidar de um doente (4). De tal modo, a prática do cuidado reflete-se na saúde e, consequentemente, na qualidade de vida (5).

0 acidente vascular encefálico (AVE) e o trauma raquimedular (TRM) são doenças neurológicas crônicas, de alta incidência, relacionadas à ocorrência de disfunções que podem gerar limitações funcionais e emocionais aos pacientes (2). Assim, esses pacientes, de uma forma ou de outra, se tornam dependentes de cuidadores, os quais nem sempre estão devidamente orientados sobre como exercer a nova função, porque na maioria das vezes são incumbidos a essa condição de uma forma inesperada (6). Porém, destaca-se cada vez mais a importância do trabalho desses cuidadores para a recuperação e melhora da qualidade de vida dessas pessoas que necessitam de acompanhamento frequente (7). 
Nesse contexto, o objetivo desta pesquisa foi avaliar a influência de uma abordagem com orientações fisioterapêuticas na qualidade de vida e na sobrecarga de cuidadores informais de pacientes com disfunções decorrentes de TRM ou AVE.

\section{Materiais e métodos}

A amostra da pesquisa foi composta por cuidadores informais de pessoas com disfunções decorrentes de AVE ou TRM que estavam em atendimento na Clínica-Escola de Fisioterapia da Faculdade AngloAmericano, em Foz do Iguaçu, Paraná.

Foram considerados como critérios de inclusão: ser familiar ou responsável com idade igual ou superior a 18 anos; e de exclusão foram: famílias nas quais não foi possível identificar o cuidador principal, e aqueles com dificuldade para comunicação verbal ou escrita.

Foram identificados 20 cuidadores, 16 do sexo feminino e 4 do masculino, com idade de 21 a 69 anos. No entanto, 8 não compareceram aos encontros agendados por não terem condução ou por não terem licença para ausentar-se do trabalho. Dessa forma, a amostra foi composta por 12 cuidadores, todos do sexo feminino, com idade média de $50,5 \pm 16,5$ anos, sendo 4 cuidadores de pacientes portadores de TRM e 8 de AVE.

No primeiro encontro, os cuidadores receberam informações sobre a participação no estudo e assinaram o Termo de Consentimento Livre e Esclarecido de acordo com a Resolução n. 196/96 do Conselho Nacional de Saúde. A pesquisa foi aprovada pelo Comitê de Ética em Pesquisa da Faculdade Assis Gurgacz (CEP/FAG), Parecer n. 063/2011. Os cuidadores receberam uma caderneta e foram instruídos a anotar as dúvidas e dificuldades encontradas no dia a dia, tanto as suas como as do paciente. Após uma semana, retornaram e devolveram as cadernetas com as anotações. Nesse dia também foram submetidos a uma avaliação da qualidade de vida e da sobrecarga.

A qualidade de vida foi avaliada utilizando o questionário Short-Form Survey, conhecido como SF-36. É um instrumento composto por 36 questões, e consiste em 8 domínios, com escores que variam de 0 a 100 , considerando a percepção do paciente sobre a forma como sua saúde e seu bem-estar e a maneira como desempenha suas atividades de vida diária interferem em sua qualidade de vida (8).

Para avaliar a sobrecarga, foi utilizado o Questionário de Avaliação da Sobrecarga do Cuidador
Informal (QASCI). O QASCI destina-se a avaliar sobrecarga física, emocional e social dos cuidadores informais. Inclui 32 itens avaliados por uma escala ordinal de frequência que varia de 1 a 5 , sendo o índice e a sobrecarga diretamente proporcionais. Integra sete dimensões: sobrecarga emocional (4 itens); implicações na vida pessoal do cuidador (11 itens); sobrecarga financeira ( 2 itens); reações a exigências (5 itens); percepção dos mecanismos de eficácia e de controle (3 itens); suporte familiar (2 itens); e satisfação com o papel e com o familiar (5 itens) (9).

Todos os instrumentos de avaliação foram autoaplicáveis. Duas semanas após a entrega das cadernetas e as avaliações da qualidade de vida e sobrecarga, foi realizado um novo encontro. Neste, os cuidadores participaram de uma aula teórico-prática e também receberam um manual de orientações. Todas essas atividades foram baseadas nas orientações do Guia Prático do Cuidador (10), nas anotações da caderneta e nas respostas do QASCI e do SF-36 e objetivaram esclarecer e orientá-los para a prática dos cuidados diários. Após 4 semanas, os cuidadores foram reavaliados utilizando os mesmos instrumentos da avaliação inicial.

Foi realizada análise estatística descritiva para calcular a média e o desvio padrão dos resultados do questionário SF-36 e as medianas do QASCI. Para a comparação pré e pós-intervenção dos questionários, empregou-se o teste de normalidade KolmogorovSmirnov e a distribuição foi considerada normal se $p>0,05$. Como todas as variáveis apresentaram distribuição normal, utilizou-se o teste $t$ pareado para a detecção de diferenças entre os resultados. 0 nível de significância foi estabelecido em $\mathrm{p} \leq 0,05$. Os testes foram realizados com o uso do software Graphpad Instat versão 3.0.

\section{Resultados}

A caracterização da amostra de cuidadores informais está demonstrada no Quadro 1.

Em relação às anotações feitas pelos cuidadores, as dúvidas estavam relacionadas ao cuidado específico com o paciente e com a própria saúde física e mental. A maior dificuldade no cuidado estava relacionada ao banho. Enquanto as queixas apresentadas foram: dor nas costas, dificuldade para conseguir dormir, falta de ânimo, discussões com o paciente, tristeza nos dias em que o paciente não se encontra bem. 
Também foram citados pontos positivos: felicidade em sair para um passeio junto do paciente, felicidade por paciente ter caminhado.

Na avaliação da qualidade de vida, com o questionário SF-36, não se observou incremento no índice geral da qualidade de vida, tendo como escore inicial $52,45 \pm 25,59$ e após a intervenção $57,03 \pm 25,42$ ( $\mathrm{p}=0,1226)$. Em ralação aos demais componentes e domínios do SF-36, apenas para os domínios dor e saúde mental observou-se diferença significativa após as orientações (Quadro 2).

Na avaliação da sobrecarga do cuidador informal, por meio do QASCI, não se observou diferença após as orientações fisioterapêuticas (Quadro 3).

\begin{tabular}{|c|c|c|}
\hline Características & $\mathbf{n}$ & $\%$ \\
\hline \multicolumn{3}{|l|}{ Sexo } \\
\hline Feminino & 12 & $100 \%$ \\
\hline \multicolumn{3}{|l|}{ Idade (anos) } \\
\hline $20-30$ & 2 & $17 \%$ \\
\hline $31-40$ & 2 & $17 \%$ \\
\hline $41-50$ & 1 & $8 \%$ \\
\hline $51-60$ & 2 & $17 \%$ \\
\hline $61-70$ & 5 & $42 \%$ \\
\hline \multicolumn{3}{|l|}{ Escolaridade } \\
\hline Ensino Fundamental Incompleto & 1 & $8 \%$ \\
\hline Ensino Fundamental Completo & 4 & $33 \%$ \\
\hline Ensino Médio Incompleto & 2 & $17 \%$ \\
\hline Ensino Médio Completo & 2 & $17 \%$ \\
\hline Superior Incompleto & 1 & $8 \%$ \\
\hline Superior Completo & 1 & $8 \%$ \\
\hline Pós-graduação & 1 & $8 \%$ \\
\hline
\end{tabular}

Quadro 1 - Caracterização da amostra

(Conclusão)

\begin{tabular}{lcc}
\hline Características & $\mathbf{n}$ & $\%$ \\
\hline Renda familiar (salários mínimos) & & \\
\hline Até 2 & 8 & $67 \%$ \\
De 3 a 4 & 3 & $25 \%$ \\
Acima de 5 & 1 & $8 \%$ \\
\hline Tempo que cuida (anos) & & \\
\hline Menos de 1 & 1 & $8 \%$ \\
De 1 a 3 & 3 & $25 \%$ \\
De 3 a 5 & 6 & $50 \%$ \\
De 5 a 10 & 1 & $8 \%$ \\
Mais de 10 & & \\
\hline
\end{tabular}

\section{Parentesco}

\begin{tabular}{lcc}
\hline Esposa & 6 & $50 \%$ \\
Mãe & 2 & $17 \%$ \\
Filha & 3 & $25 \%$ \\
Irmã & 1 & $8 \%$ \\
\hline
\end{tabular}

\section{Cuida 0 dia todo}

\begin{tabular}{lll}
\hline Sim & 9 & $75 \%$ \\
Não & 3 & $25 \%$ \\
\hline
\end{tabular}

Dias que cuida por semana

\begin{tabular}{lcc}
\hline Todos os dias & 12 & $100 \%$ \\
\hline Patologia do paciente & & \\
\hline AVE & 8 & $67 \%$ \\
TRM & 4 & $33 \%$ \\
\hline
\end{tabular}

Fonte: Dados da pesquisa. 
Quadro 2 - Qualidade de vida pré e pós-orientação (escores do SF-36, média \pm desvio padrão)

\begin{tabular}{lccc}
\hline & Pré & Pós & p \\
\hline Componente físico & $55,25 \pm 24,53$ & $62,33 \pm 23,74$ & 0,1334 \\
Capacidade funcional & $69,17 \pm 22,95$ & $76,67 \pm 10,73$ & 0,2496 \\
Aspectos físicos & $43,75 \pm 30,39$ & $43,75 \pm 35,56$ & 0,9999 \\
Dor & $51,67 \pm 17,82$ & $65,58 \pm 16,43$ & $0,0316^{*}$ \\
Estado geral de saúde & $56,42 \pm 20,77$ & $63,33 \pm 12,01$ & 0,2542 \\
\hline Componente mental & $49,64 \pm 26,58$ & $51,72 \pm 26,18$ & 0,5694 \\
Vitalidade & $48,75 \pm 15,09$ & $51,67 \pm 16,00$ & 0,3767 \\
Aspectos sociais & $52,50 \pm 26,13$ & $53,13 \pm 16,96$ & 0,9296 \\
Aspectos emocionais & $41,64 \pm 40,50$ & $36,09 \pm 38,80$ & 0,6578 \\
Saúde mental & $55,67 \pm 18,64$ & $66,00 \pm 19,71$ & $0,0029^{* *}$ \\
\hline
\end{tabular}

Legenda: ${ }^{*}=$ significativo; ${ }^{*}=$ muito significativo.

Fonte: Dados da pesquisa.

Quadro 3 - Sobrecarga do cuidador pré e pós-orientação (índices do QASCI, mediana)

\begin{tabular}{lccc}
\hline & Pré & Pós & p \\
\hline Sobrecarga emocional (4-20) & 11 & 9 & 0,0636 \\
Implicações na vida pessoal (11-55) & 31,5 & 22 & 0,0514 \\
Sobrecarga financeira (2-10) & 4 & 4 & 0,1212 \\
Reações a exigências (5-25) & 11,5 & 9,5 & 0,0963 \\
Mecanismos de eficácia e de controle (3-15) & 12,5 & 14 & 0,0745 \\
Suporte familiar (2-10) & 8,5 & 9,5 & 0,1184 \\
Satisfação com o papel de cuidador e com o familiar (5-25) & 23,5 & 22 & 0,8056 \\
\hline
\end{tabular}

Fonte: Dados da pesquisa.

\section{Discussão}

Sabe-se que não só os pacientes, mas também seus cuidadores precisam de atenção e apoio profissional (11), pois, orientar os cuidadores quanto à saúde e atividades de vida diária traz benefícios à recuperação do paciente e ainda proporciona maior tranquilidade e apoio aos que desempenham a difícil tarefa de cuidar. Portanto, pode-se considerar essencial um treinamento que assegure um cuidado eficaz e de qualidade. Porém, é necessária uma supervisão da efetividade do cuidado realizado para facilitar o ato de cuidar e colaborar no desenvolvimento dessas habilidades. Assim, é possível promover um cuidado livre do desgaste, uma vez que, dominando as ações necessárias na promoção do cuidado, o cuidador terá mais facilidade no planejamento do seu cotidiano, tendo maior tempo para proceder com o seu autocuidado $(12,13)$.

Nesse contexto, a família tem uma participação importante, com destaque para as mulheres, que, mesmo com todas as alterações estruturais ocorridas na família nas últimas décadas, continuam sendo as principais responsáveis pelo cuidado de familiares com algum tipo de incapacidade (9). No presente estudo, foi observada a exclusividade de familiares e de mulheres que eram as principais responsáveis pelo 
cuidado; sendo metade delas, esposas. Fato semelhante a outras pesquisas, que também observaram um predomínio de esposas cuidadoras $(14,15,16,17)$.

0 papel da família cuidadora é dar suporte e apoio emocional, contribuindo para a recuperação do familiar acometido pela doença e, na maioria das vezes, são essas mesmas pessoas que assumem a responsabilidade de dar continuidade à assistência, quando o doente retorna ao domicilio (18). Portanto, além da necessidade de aprender novas habilidades para lidar com o paciente acometido por algum tipo de patologia, a família necessita de apoio psicológico para superar inúmeras situações específicas como: restrições do paciente, imprevisibilidade do prognóstico, cuidados básicos como alimentação e higiene, entre outros (19). De forma positiva, essas novas habilidades para exercer o cuidado permitem que o familiar se torne parceiro dos serviços de saúde (20).

Em contrapartida, essa nova atribuição gera uma sobrecarga emocional e física, fatores que implicam em redução na qualidade de vida de cuidadores informais $(2,17,21)$. Além disso, a experiência de cuidar é multidimensional, apresentando vários fatores que podem ou não influenciar na qualidade de vida dos cuidadores (15), gerando distintas formas de compreensão do cuidado (22).

Em relação à parte física, todos os domínios avaliados apresentaram-se alterados. Sabe-se que a presença de dor influencia nos demais domínios (capacidade funcional, aspectos físicos e estado geral de saúde), pois a dor é o indicador mais relacionado com as incapacidades $(23,24)$. Mais de $70 \%$ dos cuidadores de pessoas que necessitam de assistência para transferência queixam-se de dor lombar (25); os cuidadores que apresentam maior nível de sobrecarga também apresentam mais riscos para lesões, além disso, a maioria dos cuidadores que referiram alguma lesão a atribuem à prestação do cuidado (26).

Já em relação ao componente mental, também se observou um baixo escore para todos os domínios (vitalidade, aspectos sociais, aspectos emocionais e saúde mental). Sugere-se como possíveis problemas o cansaço e os distúrbios do sono, pelo fato de dormirem tarde e terem seus sonos interrompidos para oferecerem cuidados (27). Outro fator observado é a privação de atividades de lazer, acarretando entre os cuidadores diminuição do convívio social (28); observa-se também cansaço e frustração diante da rotina, uma vez que os cuidadores não obtêm resultado efetivo na recuperação (29).
A satisfação com a vida, apesar de associada à incapacidade em condições crônicas de saúde, parece não estar relacionada a desfechos funcionais, entretanto, muitos cuidadores declaram-se insatisfeitos com a vida (23); assim, uma baixa satisfação com a vida pode ou não gerar incapacidade para o cuidador (30). Entre os principais sentimentos verbalizados pelos cuidadores destacam-se: desapego, desinteresse, culpa, desespero, raiva (31), solidão, carinho, tristeza, irritação, desânimo, revolta, insegurança, negativismo e medo. Mas, também são citados sentimentos antagônicos: amor e raiva, paciência e intolerância (32), ou seja, cuidar de um familiar é uma tarefa complexa e singular, gerando uma ambivalência de sentimentos (22).

Também é possível afirmar que, quanto maior o grau de dependência do doente, maiores serão suas dificuldades no papel que o cuidador desempenha, implicando em crescente sobrecarga física, emocional, social e financeira (33). Acrescente-se que algumas doenças provocam sinais e sintomas que também podem interferir na saúde do cuidador (21). Outro fator apontado é a saúde mental do paciente, pois cuidadores de pacientes com transtornos mentais apresentam uma maior sobrecarga quando comparados aos cuidadores de pacientes com distúrbios somatossensoriais (34); cabe esclarecer, porém, que, na presente pesquisa, o foco foi a saúde do cuidador, não sendo avaliadas as disfunções do paciente; por outros termos, não foi estabelecida uma relação entre a saúde mental dos pacientes com uma possível sobrecarga no cuidado.

Apesar dessa inter-relação e multidimensionalidade entre os domínios saúde física e mental, a intervenção proposta só repercutiu de forma significativa nos domínios dor e saúde mental.

Com relação à caracterização da amostra, os dados demonstraram que, com relação à idade, os dados obtidos são semelhantes a outras pesquisas $(14,15$, $16,17)$, tendo as cuidadoras idade média entre 50 e 60 anos de idade. Como também um predomínio de cuidadoras com Ensino Fundamental e Médio (16), além de referirem baixa renda familiar (21).

A inserção de cuidadores informais de pacientes que estavam em atendimento fisioterapêutico como objeto deste estudo foi uma proposta de intervenção que visava tanto o cuidado do cuidador, quanto de quem é cuidado, pois essas são estratégias promissoras para melhorar a qualidade de vida dos cuidadores (14). As orientações repassadas foram baseadas nas dificuldades e dúvidas, do cuidador e do paciente, relatadas no período de uma semana. Tal 
procedimento objetivou conhecer as necessidades das pessoas para tentar saná-las. Sabe-se que é de fundamental importância conhecer a realidade dos cuidadores, porque dessa forma é possível promover ações integrais em saúde que contemplem soluções para minimizar os efeitos danosos da sobrecarga do cuidado vivenciada por eles (35).

Os resultados apresentados se limitam a uma amostra pequena de cuidadores, formada por demanda espontânea. Além disso, o estudo se propôs a avaliar, a curto prazo, os efeitos de uma única intervenção fisioterapêutica, baseada em orientações teórico-práticas e no fornecimento de um material de apoio com as mesmas orientações. Porém, não se averiguou a execução das orientações e nem foi mensurada a quantidade de informação assimilada. Assim, sugere-se que estudos de seguimentos sejam desenvolvidos, incluindo maior frequência de encontros, acompanhamento e avaliação do treinamento dos cuidadores, além de uma abordagem interdisciplinar.

\section{Considerações Finais}

Uma intervenção com orientações fisioterapêuticas teórico-práticas repercutiu de forma positiva para redução da dor e melhora da saúde mental em cuidadores informais de pessoas com disfunções decorrentes de AVE ou TRM.

\section{Referências}

1. Brasil. Ministério do Trabalho e Emprego. Classificação brasileira de ocupações [citado 30 jan. 2013]. Disponível em: http://www.mtecbo.gov.br.

2. Sousa AG, Zarameli RC, Ferrari RAM, Frigero M. Avaliação da qualidade de vida de cuidadores de pacientes com seqüelas neurológicas. ConScientiae Saúde. 2008;7(4):497-502.

3. Perlini MNOG, Faro ACM. Cuidar de pessoa incapacitada por acidente vascular cerebral no domicílio: o fazer do cuidador familiar. Rev Esc Enferm-USP. 2005;39(2):15463. doi:10.1590/S0080- 62342005000200005.

4. Dillehay RC, Sandys MR. Caregivers for Alzheimer's patients: what we are learning from research. Int J Aging Hum Dev. 1990;30(4):263-85. doi:10.2190/ 2P3J-A9AH-HHF4-00RG.
5. Trigueiro LCL, Lucena NMG, Aragão POR, Lemos MTM. Perfil sociodemográfico e índice de qualidade de vida de cuidadores de pessoas com deficiência física. Rev Fisioter Pesq. 2011;18(3):223-7.

6. Foss MHD, Martins MRI, Mazaro LM, Martins MID, Godoy JMP. Qualidade de vida dos cuidadores de amputados de membros inferiores. Rev Neuroc. 2009; 17(1):8-13.

7. Masson LP, Brito JC, Sousa RNP. 0 trabalho e a saúde de cuidadores de adolescentes com deficiência: uma aproximação a partir do ponto de vista da atividade. Saúde Soc. 2008;17(4):68-80.

8. Ciconelli RM, Ferraz MB, Santos W, Meinão I, Quaresma RM. Tradução para o português e validação do questionário genérico de avaliação de qualidade de vida SF-36. Rev Bras Reumatol. 1999;39(3):580-8.

9. Martins T, Ribeiro JP, Garrett C. Estudo de validação do questionário de avaliação da sobrecarga para cuidadores informais. Psico Saúde Doença. 2003;4(1):131-48

10. Brasil. Ministério da Saúde. Guia prático do cuidador. 2008. Disponível em: http://bvsms.saude.gov.br/bvs/ publicacoes/guia_pratico_cuidador.pdf.

11. Opara JA, Jaracz K. Quality of life of post-stroke patients and their caregivers. J Med Life. 2010;3(3):216-20.

12. Lavinsky AR, Vieira TT. Processo de cuidar de idosos com acidente vascular encefálico: sentimentos dos familiares envolvidos. Acta Sci, Health Sci. 2004; 26(1):41-5.

13. Bicalho CS, Lacerda MR, Catafesta F. Refletindo sobre quem é o cuidador familiar. Cogitare Enferm. 2008; 13(1):118-23.

14. Schulz R, Czaja SJ, Lustig A, Zdaniuk B, Martire LM, Perdomo D. Improving the quality of life of caregivers of persons with spinal cord injury: a randomized controlled trial. Rehabil Psychol. 2009;54(1):1-15. doi: $10.1037 / \mathrm{a} 0014932$.

15. Van Puymbroeck M, Rittman MR. Quality-of-life for predictors for caregivers at 1 and 6 months poststroke: results of path analyses. J Rehabil Res Dev. 2005; 42(6):747-60. doi:10.1682/JRRD.2005.01.0025.

16. Cramm JM, Strating MMH, Nieboer AP. Validation of the caregivers' satisfaction with stroke care questionnaire: C-SASC hospital scale. J Neurol. 2011;258:100812. doi:10.1007/s00415-010-5871-2. 
17. Makiyama TY, Battisttella LR, Litvoc J, Martins LC. Estudo sobre a qualidade de vida de pacientes hemiplégicos por acidente vascular cerebral e de seus cuidadores. Acta Fisiatr 2004;11(3):106-9.

18. Alvarez AM. Tendo de cuidar: a vivência do idoso e sua família cuidadora no processo de cuidar e ser cuidado em contexto domiciliar [tese]. Florianópolis: Universidade Federal de Santa Catarina; 2001.

19. Rowland LP. Tratado de neurologia. 12. ed. Rio de Janeiro: Guanabara Koogan; 2011.

20. Alonso, ILK. Luzes e sombra no ritual do encontro entre o universo profissional e o mundo da intimidade familiar: a intervenção profissional na saúde da família em âmbito domiciliar [tese]. Florianópolis: Universidade Federal de Santa Catarina; 2003.

21. Moreira PHB, Mafra SCT, Pereira ET, Silva VE. Qualidade de vida de cuidadores de idosos vinculados ao Programa Saúde da Família - Teixiera, MG. Rev Bras Geriatr Gerontol. 2011;14(3):433-40. doi:10.1590/ S1809-98232011000300004.

22. Mendonça FF, Garanhani ML, Martins VL. Cuidador familiar de sequelados de acidente vascular cerebral: significado e implicações. Physis. 2008;18(1):143-58.

23. Maia AC, Fialho CB, Alcântara MA, Morais RLS. Incapacidade funcional associada à lombalgia em cuidadores de crianças com paralisia cerebral grave. Rev Fisioter Pesq. 2008;15(4):349-54. doi:10.1590/ S1809-29502008000400006.

24. Gheldof ML, Vinck J, Bussche VE, Vlaeyen SWJ, Hidding A, Crombez G. Pain and pain-related fear are associated with functional and social disability in an occupation setting: evidence of mediation by pain-related fear. Eur J Pain. 2006;10:513-25. doi:10.1016/j. ejpain.2005.07.005.

25. Tong HC, Haig AJ, Nelson VS, Yamakawa KSJ, Kandala G, Shin KY. Low-back pain in adult female caregivers of children with physical disabilities. Arch Pediatr Adolesc Med. 2003;157:1128-33. doi:10.1001/ archpedi.157.11.1128.

26. Hayes J, Chapman P, Young LJ, Rittman M. The prevalence of injury for stroke caregivers and associated risk factors. Top Strokes Rehabil. 2009;16(4):300-7. doi:10.1310/tsr1604-300.
27. Williams AM. Caregivers of person with stroke: their physical and emotional well being. Qual Life Res. 1993;2(3):213-20. doi:10.1007/BF00435225.

28. Cattani RB, Girardon-Perlini NMO. Cuidar do idoso doente no domicilio na voz de cuidadores familiares. Rev Eletr Enf. 2004;6(2):254-71.

29. Andrade OG, Rodrigues RAP. O cuidado familiar ao idoso com sequela de acidente vascular cerebral. Rev Gaúcha Enferm. 1999;20(2):90-109. PMid:11998109.

30. Kovacs MF, Abraira V, Zamora J, Teresa Gil Del Real M, Llobera J, Fernandéz C, et al. Correlation between pain, disability, and quality of life in patients with common low-back pain. Spine. 2004;29(2):206-10. doi:10.1097/01.BRS.0000107235.47465.08.

31. Martins T, Ribeiro JP, Garrett C. Questionário de avaliação da sobrecarga do cuidador informal (QASCI) - reavaliação das propriedades psicométricas. Referência. 2004;11:17-31.

32. Silveira TM, Caldas CP, Carneiro TF. Cuidando de idosos altamente dependentes na comunidade: um estudo sobre cuidadores familiares principais. Cad Saúde Pública. 2006;22(8):1629-38. doi:10.1590/ S0102-311X2006000800011.

33. Abreu EP, Ramos SIV. O regresso a casa do doente vértebro-medular: o papel do cuidador informal. 2007 [citado 20 jan 2012]. Disponível em: http://www.psicologia.pt/artigos/textos/A0366.pdf.

34. Haustrup LH, Van Den Berg B, Gyrd-Hansen D. Do informal caregivers in mental illness feel more burdened? A comparative study of mental versus somatic illnesses. Scand J Public Health. 2011;39(6):598-607. doi:10.1177/1403494811414247.

35. Pinto MF, Barbosa DA, Ferreti CEL, Souza LF, Fram DS, Belasco AGS. Qualidade de vida de cuidadores de idosos com doença de Alzheimer. Acta Paul Enferm. 2009;22(5):652-7.

Recebido: 11/04/2012 Received: 04/11/2012

Aprovado: 27/12/2012 Approved: $12 / 27 / 2012$ 